\title{
Disappearing refuges in time and space \\ How environmental change threatens species coexistence
}

\author{
Journal Article \\ Author(s): \\ Münkemüller, Tamara; Reineking, Björn; Travis, Justin; Bugmann, Harald; Johst, Karin \\ Publication date: \\ 2009 \\ Permanent link: \\ https://doi.org/10.3929/ethz-b-000156868 \\ Rights / license: \\ In Copyright - Non-Commercial Use Permitted \\ Originally published in: \\ Theoretical Ecology 2(4), https://doi.org/10.1007/s12080-009-0043-7
}




\title{
Disappearing refuges in time and space: how environmental change threatens species coexistence
}

\author{
Tamara Münkemüller • Björn Reineking • \\ Justin Travis • Harald Bugmann • Karin Johst
}

Received: 24 October 2008 /Accepted: 1 February 2009/Published online: 21 February 2009

(C) Springer Science + Business Media B.V. 2009

\begin{abstract}
Understanding the impacts of environmental changes on species survival is a major challenge in ecological research, especially when shifting from singleto multispecies foci. Here, we apply a spatially explicit twospecies simulation model to analyze the effects of geographic range shifting and habitat isolation on different coexistence mechanisms. The model explicitly considers dispersal, local competition, and growth on a single resource. Results highlight that both range shifting and habitat isolation severely impact coexistence. However, the strength of these impacts depends on the underlying coexistence mechanisms. Neutrally coexisting species are particularly sensitive to habitat isolation, while stabilized coexistence through overcompensatory density regulation is much more sensitive to range shifting. We conclude that, at the community level, the response to environmental change
\end{abstract}

T. Münkemüller $(\bowtie) \cdot \mathrm{K}$. Johst

Department of Ecological Modelling,

Helmholtz Center for Environmental Research-UFZ,

Permoserstr. 15,

04301 Leipzig, Germany

e-mail: tamara.muenkemueller@ufz.de

T. Münkemüller $\cdot$ B. Reineking $\cdot H$. Bugmann

Forest Ecology, Department of Environmental Sciences,

ETH Zurich,

8092 Zurich, Switzerland

B. Reineking

Biogeographical Modelling, BayCEER,

University of Bayreuth,

Universitätsstr. 3095447 Bayreuth, Germany

J. Travis

Zoology Building, School of Biological Sciences,

University of Aberdeen,

Tillydrone Avenue,

Aberdeen AB24 2TZ Scotland, UK sensitively depends on the underlying coexistence mechanisms. This suggests that predictions and management recommendations should consider differences between neutral versus stabilized community structures whenever possible.

Keywords Climate change - Fragmentation .

Overcompensation $\cdot$ Neutral coexistence .

Species interactions $\cdot$ Range shift

\section{Introduction}

Landscape fragmentation and climate change have been identified to be among the most severe causes of global population decline and species extinction (Sala et al. 2000; Thomas et al. 2004). A substantial body of work has been devoted to understanding the potential impact of these environmental factors on species' ranges (e.g., Araujo and Rahbek 2006; Thuiller et al. 2006; Hatfield and LeBuhn 2007; Kruess and Tscharntke 1994). The great majority of this work utilizes correlational approaches to relate current distributions to current climate and then projects future distributions onto future climate (Thuiller et al. 2004; Araujo et al. 2004). However, there is an increasing recognition that, on their own, these well-established methods are insufficient for predicting future patterns of biodiversity and that a deeper ecological understanding of the process of range shifting is required (Guisan and Thuiller 2005; Heikkinen et al. 2006; Araujo and New 2007). Some progress has already been made on understanding how both the intrinsic population dynamics (Best et al. 2007) and the dispersal characteristics of a species (Higgins et al. 2003; Travis 2003; Midgley et al. 2006) determine its ability to track a changing climate, i.e., its 
ability to move from currently suitable to future-suitable areas. So far, all these models take a single-species approach. In reality, both the current distribution of a single species and its response to environmental change depend upon other species within the community. With the exception of a few rather specific examples (Davis et al. 1998; Roy et al. 2004; Ferrier and Guisan 2006; Brooker et al. 2007), there is a lack of formal modeling considering how the processes that structure a community under constant climatic conditions determine how the elements of that community will respond to climate change.

In this paper, we address these issues by analyzing coexistence of two interacting species under the increasing pressure of habitat isolation and geographic range shifting as it may be caused by climate change. We explicitly focus on whether and to what extent different coexistence mechanisms are differentially affected by these factors.

Coexistence mechanisms can be broadly classified as either stabilizing or equalizing (Chesson 2000; Adler et al. 2007). Stabilizing mechanisms rely on increased intraspecific compared to interspecific competition which relatively favors less abundant compared to more abundant species and thus facilitates the recovery of populations from low densities. Equalizing mechanisms of coexistence rely on minimizing differences in average fitness. The debate over the relative importance of stabilizing and equalizing mechanisms in different communities is ongoing. Recently, Adler et al. (2007) argued that there is no clear-cut answer to the question of which mechanism structures a community. Rather, there are gradual transitions where either strong stabilizing mechanisms overcome large fitness differences, or weak stabilization is coupled with similar fitness between species (cf. Gravel et al. 2006; Chesson 2000). For our analyses, we exemplarily chose one route to coexistence for each type of mechanism, respectively: (1) coexistence through overcompensating density regulation as a particular stabilizing mechanism where species differ only in one trait, namely their mechanism of density regulation, and (2) neutral coexistence of species with almost identical fitness and no stabilization. Below, we outline how each of these two example mechanisms theoretically operates under stable environments and indicate where they may be important in structuring real communities.

1. Coexistence through overcompensation facilitates coexistence of two species on a single resource and results from a differentiation along an axis of density regulation mechanisms, from overcompensation towards undercompensation (Münkemüller et al., submitted manuscript). With overcompensatory density regulation, populations can exhibit cyclic or chaotic dynamics whereas compensatory or undercompensatory density regulation leads to equilibrium dynamics (Münkemüller and Johst 2006).
The interaction between two species, one an overcompensator and another with sufficiently different density regulation, results in temporally alternating community dynamics that allow recovery from low densities for both species (Münkemüller et al., submitted manuscript). In field studies, it is a challenging task to identify communities that rely (fully or partly) on this coexistence mechanism because the mechanism of density regulation is particularly difficult to estimate from field data (Godfray et al. 1990; Morris 1990). However, species that exert density regulation in early life stages (and thus are most probable to overcompensate, Sinclair 1989) and live in highly diverse communities with apparently limited number of resources may depend critically on this mechanism. Examples may include the highly diverse small mammal and insect communities in some tropical rainforests where many species with no apparent differentiation in physiological characteristics or resource requirements coexist or highly diverse marine plankton communities that coexist on only a few potentially limiting resources (Huisman and Weissing 1999).

2. Species that are equal in all aspects and compete with each other in a homogeneous landscape randomly drift to extinction (Gause 1969). According to Hubbell's neutral theory of biodiversity, this drift can be slow enough to maintain coexistence over very long time periods, particularly in situations with limited dispersal (Hubbell 2001). Hubbell's theory has been successful at reproducing empirically observed patterns of species richness (cf. Rosindell and Cornell 2007; e.g., tree diversity in tropical rain forests, Hubbell 2001; Hubbell 2006; species abundance distributions in fynbos, Latimer et al. 2005; zooplankton diversity, Walker and Cyr 2007; and fish diversity, Etienne and Olff 2005; Walker and Cyr 2007) but has nevertheless been the subject of considerable debate as communities are very unlikely to be truly neutral (Gotelli and Mccabe 2002; Bell 2005; Walker 2007). Gotelli and Rohde (2002) suggested that presence-absence patterns for smallbodied taxa with low vagility and/or small populations (e.g., marine ectoparasites and herps) are mostly random, whereas those for large-bodied taxa with high vagility and/or large populations (e.g., birds and mammals) are highly structured.

In this study, we utilize a spatially explicit two-species metapopulation model to examine the impacts of habitat isolation and geographic range shifting due to climate change. We address three major research questions. First, what is the impact of dispersal limitation and resulting patch isolation (called patch isolation in the following) on the relative importance of neutral coexistence and stabilized 
coexistence in a fragmented but temporally stable landscape? Second, how do patch isolation and climate-induced range shifting - in isolation and combined-impair species assemblages? Third, do the two considered coexistence mechanisms respond differentially to changes in these environmental factors?

\section{Model description and analysis}

\section{Model description}

\section{Purpose and structure}

The model's purpose is to demonstrate effects of patch isolation and range shifting on coexistence in a two-species metapopulation. Species differ only in their density regulation. The model considers a landscape represented as a rectangular grid with habitat patches that are surrounded by matrix cells. It explicitly simulates the population dynamics of the two species in the habitat patches and models the dispersal of individuals between the patches. In each time step, local population dynamics with intraspecific and interspecific interactions are followed by dispersal.

\section{Local population dynamics}

Local population dynamics in each patch $i$ are described by an extended version of the equation by Maynard Smith and Slatkin (1973; see also Hassell and Comins 1976):

$$
N_{i, s}(t+1) \sim \operatorname{Pois}\left(\frac{N_{i, s}(t) \cdot R_{\mathrm{sp}}}{\left(1+\left(R_{\mathrm{sp}}-1\right)\left(\frac{N_{i, \mathrm{tot}}(t)}{C C_{i}(t)}\right)^{b_{s}}\right)}\right)
$$

The population sizes on patch $i$ of species $s$ at time $t$ is given by $N_{i, s}(t)$ and the species' maximum growth rate is given by $R_{\mathrm{sp}}$ and was set to five which describes fast growth at low densities. Net population growth of both species is limited by the total number of individuals living on a patch and the current carrying capacity, $\mathrm{CC}_{i}(t)$. We assume interspecific competition to be as strong as intraspecific competition, $N_{i, \text { tot }}=N_{i, A}+N_{i, B}$. Both species differ only in their mechanism of density regulation, which is characterized by $b_{s}$ ( $b_{s}<1$ corresponds to undercompensating, $b_{s}=1$ to compensating, and $b_{s}>1$ to overcompensating density regulation). This way, a wide range of combinations of density regulation mechanisms from undercompensation to strong overcompensation is comparable. We account for demographic stochasticity by drawing random numbers from a Poisson distribution. We independently and randomly initialize populations for each species by drawing from a uniform distribution between 10 and $10+\mathrm{CC}_{i}(t)$ individuals.

\section{Dispersal}

In each time step, dispersal occurs after population growth. The population size after dispersal $N_{i}(t, d=1)$ is equal to the one prior to dispersal, $N_{i}(t, d=0)$, minus the number of emigrants, $N_{\mathrm{emi}, i}(t)$, and plus the number of immigrants. The number of emigrants per patch is drawn from a binomial distribution with $N_{i}(t, d=0)$ number of trials and emigration rate $p_{\text {emi }}$. Emigration rate is constant over space and time and equal for both species. Species do not interact during dispersal. The number of immigrants from patch $i$ to patch $j$ is drawn from a multinomial distribution with $N_{\mathrm{emi}, i}(t)$ trials and transfer probability $p_{i j}$. The matrix of transfer probabilities, with entries $p_{i j}$, describes the probability to move from patch $i$ to patch $j$. Values decrease exponentially with the Euclidean distance between patches, $D_{i j}$, measured in units of grid cells:

$p_{i j}=\frac{\exp \left(-\frac{1}{\mathrm{DD}} D_{i j}\right)}{\sum_{j} \exp \left(-\frac{1}{\mathrm{DD}} D_{i j}\right)} \cdot \exp \left(-m D_{i j}\right)$

The mean distance both species are able to disperse is defined by DD and the dispersal mortality rates are defined by $m$. The denominator scales the transfer probabilities $p_{i j}$ such that they add up to one over all $j$ in the absence of dispersal mortality (for the special case, $m=0$ ). The combined effect of DD, $p_{\text {emi }}$, and $m$ defines patch isolation; populations of species with long DD, high $p_{\text {emi }}$, and low $m$ are well connected while populations of species with short $\mathrm{DD}$, low $p_{\mathrm{emi}}$, and high $m$ are isolated.

\section{Simulation experiments}

We simulated two different scenarios: in the patch isolation scenario, we addressed our first research question (what is the impact of patch isolation on the coexistence mechanisms in a temporally stable landscape?), whereas in the range-shifting scenario we focused on the remaining two research questions (what is the impact of patch isolation and climate-induced range shifting on the coexistence mechanisms? Do the reactions of the mechanisms differ?). The two scenarios differ in patch number, temporal stability of patches, and species' dispersal abilities.

The landscape in the patch isolation scenario is a lattice of 20 by 20 cells. On the lattice two, four or eight cells are randomly chosen to host habitat patches (each patch is located on a single cell no matter what its value for the carrying capacity is). Independent of the number of patches, the global capacity of available habitat is held constant (total carrying capacity of the landscape is set to 500 individuals). Thus, local carrying capacities decrease with increasing patch number $(250,125$, or 63 individuals, cf. Table 1) and 
Table 1 Description of parameters that varied across simulation experiments, parameters that were kept constant across all simulation experiments, and output variables

\begin{tabular}{|c|c|c|c|}
\hline Symbol & Parameter & Structure or process & Values \\
\hline \multicolumn{4}{|c|}{ Parameters that varied across simulation experiments } \\
\hline$L_{\text {Size }}$ & Lattice size [cells squared] & Landscape: structure & $20 \times 20$ vs. $40 \times 240$ \\
\hline$P_{\text {Number }}$ & Patch number & Landscape: structure & $2,4,8,90$ \\
\hline $\mathrm{CC}_{i}$ & Local carrying capacities & Landscape: patch growth & $63,125,250,500$ \\
\hline$b_{s}$ & Density dependence parameter & Reproduction & {$\left[\mathrm{e}^{-2} ; \mathrm{e}^{2.4}\right]$, in steps of $\mathrm{e}^{0.1}$} \\
\hline DD & Mean dispersal distance [cells] & Dispersal & $1,2,6,12,20$ \\
\hline$M$ & Dispersal mortality rate & Dispersal & $0,0.2$ \\
\hline$p_{\text {emi }}$ & Emigration rate & Dispersal & $0.01,0.1$ \\
\hline $\mathrm{CW}_{\text {size }}$ & Climate window size [cells] & Landscape: climate change & 20,40 \\
\hline $\mathrm{CW}_{\text {speed }}$ & Climate window shift speed [cells/time step] & Landscape: climate change & $0.5,1,2,4,8$ \\
\hline \multicolumn{4}{|c|}{ Parameters that were kept constant across all simulation experiments } \\
\hline$R_{s p}$ & Growth rate of species & Reproduction & 5 \\
\hline$R_{p}$ & Growth rate of patches & Landscape: patch growth & 5 \\
\hline $\mathrm{CC}_{\max }$ & Maximum carrying capacity & Landscape: patch growth & 500 \\
\hline \multicolumn{4}{|c|}{ Output variables } \\
\hline CPR & Coexistence probability $^{\mathrm{a}}$ & & \\
\hline $\mathrm{CPO}$ & Coexistence potential $^{\mathrm{a}}$ & & \\
\hline
\end{tabular}

${ }^{\text {a }}$ Variable

are constant over time. However, increasing the number of patches decreases the Euclidean distance between patches and thus results in a mean decrease of realized dispersal mortalities. We investigated patch isolation effects by comparing different dispersal abilities: species with longdistance dispersal and no dispersal mortality ( $D D=20$ and $m=0$, cf. Table 1) and species with short-distance dispersal and severe dispersal mortality $(\mathrm{DD}=1$ and $m=0.2$, cf. Table 1). We selected these two combinations of dispersal distance and dispersal mortality to contrast the two extremes of all four possible combinations. In total, we conducted 621,000 simulations for the patch isolation scenario: three different patch numbers times two levels of patch isolation times 1,035 combinations of density regulation mechanisms (triangular matrix with diagonal and 45 different density regulation mechanisms: $45 \times 46 / 2$ ) times 100 repetitions.

The landscape in the range-shifting scenario is a lattice of 240 cells in width by 40 cells in height with 90 potential habitat patches distributed uniformly and randomly across it. Local carrying capacities are dynamic in the sense that not all 90 potential habitat patches provide resources in each time step but only those patches within a "climate window" (Travis 2003; Best et al. 2007). This climate window has a certain size, $\mathrm{CW}_{\text {size }}$, and moves across the landscape over time. The number of cells the window moves per time step is defined as the speed of range shifting, $\mathrm{CW}_{\text {speed, }}$, which is abbreviated with shift speed in the following (Fig. 1). Initially, the window is located at the left hand side of the landscape and remains stationary for the first 100 time steps. In the stationary period, all carrying capacities within the window are set to the maximum carrying capacity (patches can sustain a population), outside all carrying capacities are set to zero (patches cannot sustain a population). After the stationary period, the window starts moving to the right hand side of the landscape and stops only after reaching the border of the landscape. Potential habitats entering the window gradually become suitable for the species; potential habitats leaving the window instantly lose their suitability and local populations go extinct. The local carrying capacity of a patch, $C C_{i}(t)$, upon entering the window is set to a value of one individual and its temporal development is governed by the equation of Maynard Smith and Slatkin (1973):

$$
\mathrm{CC}_{i}(t+1)=\frac{\mathrm{CC}_{i}(t) \cdot R_{p}}{1+\left(R_{p}-1\right) \mathrm{CC}_{i}(t) / \mathrm{CC}_{\max }}
$$

The patch growth rate, $R_{p}$, determines how quickly patches become suitable once entering the climate window. We assumed fast growth $\left(R_{p}=5\right)$. The maximum carrying capacity, $\mathrm{CC}_{\max }$, was set to 500 individuals. Patch dynamics occur in each time step and are followed by local population dynamics and dispersal. We investigated geographic range shifting due to climate change via increasing shift speed $\left(\mathrm{CW}_{\text {speed }}=0.5,1,2,4,8\right)$ and different sizes of the climate window $\left(\mathrm{CW}_{\text {size }}=20\right.$ or 40 ; 


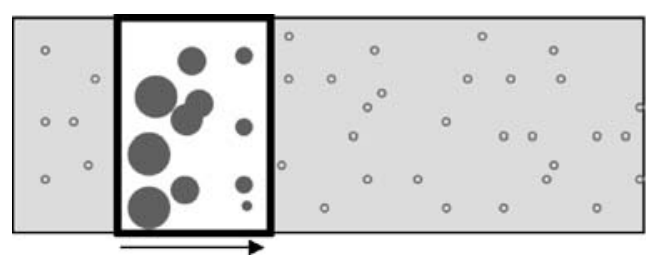

Fig. 1 Geographic range shifting modeled by a moving climate window (bold rectangular with rectangular size indicating range size). Over time, the window moves across the landscape; potential habitat patches (gray circles) gradually become suitable for the two species

cf. Table 1). We analyzed patch isolation effects comparing different dispersal distances $(\mathrm{DD}=1,2,6,12,20)$ and emigration rates $\left(p_{\text {emi }}=0.01\right.$ or 0.1 ; cf. Table 1$)$. In total, we conducted 9,030,000 simulations for the range-shifting scenario: two climate window sizes times two emigration rates times five shift speeds times five dispersal distances times 903 combinations of density regulation mechanisms times 100 repetitions.

The simulation model is implemented with the Borland $\mathrm{C}++$ Builder 5; random number distributions are taken from the GNU Scientific Library (GSL Team 1992), and graphics are created with R 2.6.1 (R Development Core Team 2007).

\section{Aggregated output variables}

To facilitate the comparison between equalizing and stabilizing mechanisms, we aggregated the information from time series of species' abundances (Fig. 2a) in two measures, the coexistence probability (CPR; Fig. 2b) and the coexistence potential (CPO; Fig. 2c).

\section{Coexistence probability}

Firstly, we aggregated 100 replications of one combination of density regulation mechanisms into an estimate of the probability for the two species to still coexist in the landscape after 1,000 years. We repeated this procedure for different combinations of density regulation mechanisms ranging from undercompensation to strong overcompensation. The species were identical in all other traits. We plotted the CPR of the possible combinations of density regulation mechanisms in a triangular matrix to illustrate the parameter window of coexistence (Fig. 2b, the gray shading codes CPR for different combinations of $b_{s}$ values).

\section{Coexistence potential}

Secondly, we aggregated the size (i.e., the number of parameter combinations) and the strength (i.e., the coexistence probability) of the parameter window of coexistence in a new output variable called coexistence potential, CPO, and assumed that both size and strength of the window

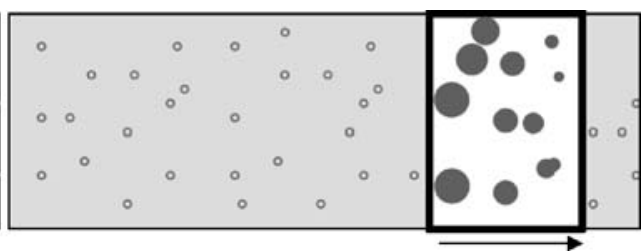

(diameters correspond to sizes of carrying capacity) and species can immigrate. Once the window has moved, past patches become unsuitable and individuals go extinct

characterized the overall likelihood of coexistence. We argue that the more parameter combinations result in coexistence and the higher the probability to coexist, the more likely it is that a favorable combination of density regulation mechanisms evolves in an ecosystem. Thus, we measured the coexistence potential, $\mathrm{CPO}$, by summing up all coexistence probabilities for all different combinations of density regulation mechanisms. We repeated this procedure for different patch isolation and range-shifting regimes (Fig. 2c). This way, we did not get an absolute measure of coexistence potential as the value depends on the analyzed ranges and number of parameter combinations but a relative measure that allows quantitatively comparing and ranking different landscape scenarios.

\section{Results}

Within our first research question, we started analyzing the distinctiveness of our aggregated measures with regard to the two coexistence mechanisms, neutral coexistence and stabilized coexistence, in temporally constant but spatially fragmented landscapes (patch isolation scenario). We plotted the coexistence probability for the different combinations of density regulation mechanisms. We found two distinct and cohesive regions of parameter combinations for density regulation that led to coexistence (Fig. 3). Coexistence probability was high either if species displayed moderate dissimilarities in their density regulation, i.e., one species with overcompensation and the other different (e.g., Fig. 3b, stabilized coexistence along the negative diagonal), or if species showed equal or very similar density regulation (e.g., Fig. 3d, neutral coexistence along the positive diagonal). Analyzing the dependency of the coexistence potential on dissimilarities in density regulation in more detail showed a distinct depression at the $b$ ratio $\left(b_{A} / b_{B}\right)$ of $\mathrm{e}^{0.6} \sim 1.82$ and local maxima below as well as above this value. Based on this result, we defined that neutral coexistence corresponded to $b$ ratios below 1.82 while stabilized coexistence corresponded to $b$ ratios above 1.82. (cf. gray vertical bar, Fig. 4a and b). Analyzing the influence of patch isolation on coexistence, we could identify distinct 


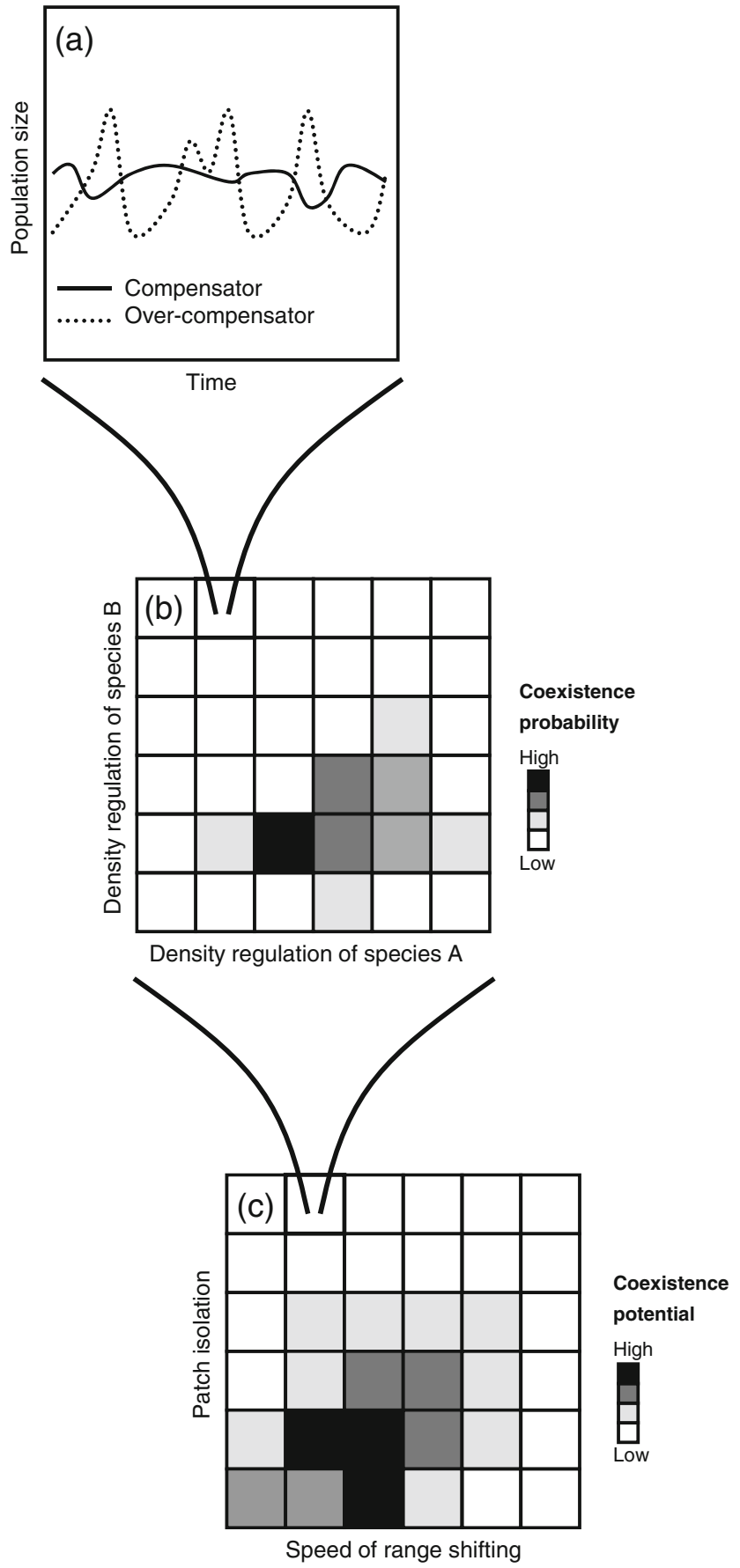

Fig. 2 Aggregation steps to derive measures for coexistence probability and coexistence potential. Panel a shows a time series of population sizes for two competing species. We repeated 100 time series for each parameter set and calculated the coexistence probability CPR, i.e., percentage of time series where both species survived at least 1,000 years as a measure of. Panel $b$ shows the values of CPR for different combinations of density regulation values. Finally, the sum of CPR for all analyzed combinations of density regulation values was used as a measure for CPO. Panel $c$ shows the values of CPO for different speeds of range shifting (shift speeds) and a number of dispersal distances differences between the two coexistence mechanisms. Stabilized coexistence was strong and robust in a landscape with two patches, no matter whether they were well connected or isolated (Fig. 3a and b). With an increasing number of patches, local carrying capacity decreased and fewer combinations of density regulation mechanisms resulted in stabilized coexistence. In contrast, neutral coexistence only occurred if patches were sufficiently isolated (Fig. 3d and f). The higher is the number of patches, the more combinations of density regulation mechanisms could coexist and the less similar species needed to be. Neither of the two mechanisms supported coexistence on eight well-connected but small patches (Fig. 3e). However, on eight isolated patches, both coexistence mechanisms could result in persistence

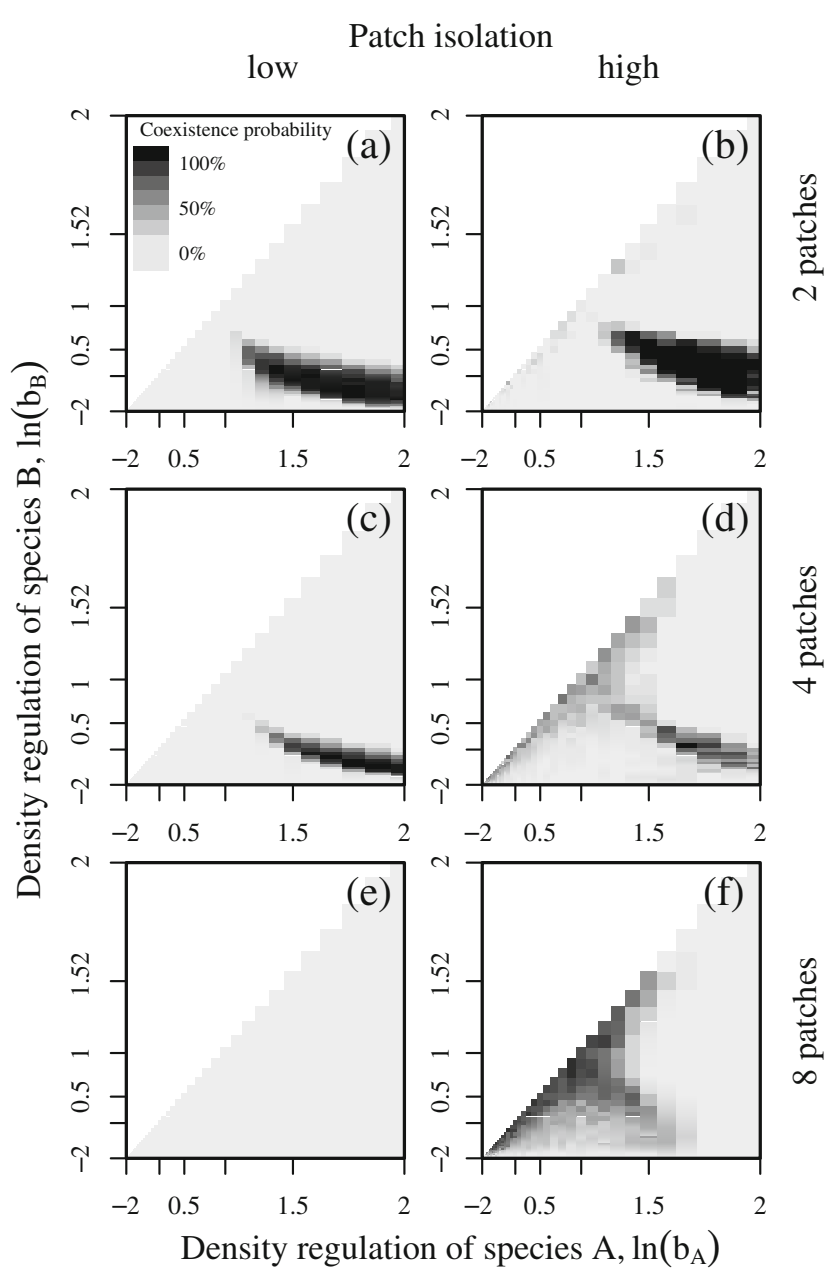

Fig. 3 Coexistence probability of two competing species for different combinations of density regulation $(\ln (b)<0$ : undercompensation, $\ln$ (b) $=0$ : compensation, $\ln (\mathrm{b})>0$ : overcompensation). Patch isolation is low in the left (mean dispersal distance 20 cells and no dispersal mortality, $m=0$, plots $a, c, e$ ) and high in the right column (mean dispersal distance one cell and severe dispersal mortality, $m=0.2$, plots $b, d, e)$. The number of patches in the landscape increases top-down from two to eight patches. Darker colors indicate higher coexistence probability, white indicates no simulations 
(Fig. 3f). In summary, stabilized coexistence dominated in landscapes with either a low number of patches (two connected or isolated patches, Fig. 4) or in landscapes with a greater number of strongly connected and moderately large patches (four connected patches, Fig. 4a and c). Neutral coexistence dominated in landscapes with many but rather isolated patches (Fig. $4 \mathrm{~b}$ and d). However, species at the intersection of stabilized coexistence and neutral coexistence were able to coexist in landscapes with four or eight isolated patches, indicating that here both mechanisms acted in concert to facilitate coexistence.

Within our second research question, we investigated how patch isolation and range shifting impaired species assemblages both separately and in combination (rangeshifting scenario). In general, increasing shift speed as well as increasing patch isolation (through reduced dispersal distances) strongly decreased coexistence (Fig. 5). Severe impacts on coexistence occurred even under very moderate shift speeds. Further increasing the shift speed did result in a greater impact but most of the damage was already done at slow range shifting. For increased patch isolation, an intermediate range of dispersal distances was most sensitive to a further decrease. Species with low emigration rates and narrow climate windows were most vulnerable to range shifting and patch isolation such that already very slow

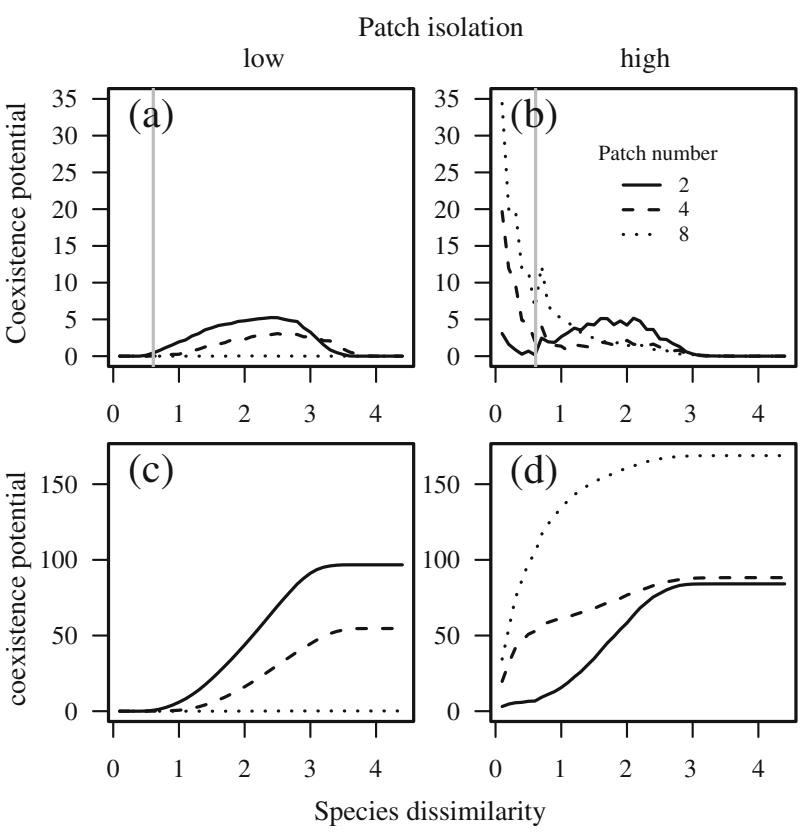

Fig. 4 Coexistence potential in dependency on species dissimilarity for different patch isolation. Patch isolation is low in the left (mean dispersal distance 20 cells and no dispersal mortality, $m=0$, plots $a, c$ ) and high in the right column (mean dispersal distance one cell and severe dispersal mortality, $m=0.2$, plots $b, d$ ). Gray vertical lines indicate the selected criterion for separation of the two coexistence mechanisms (only shown in the non-cumulative plots)

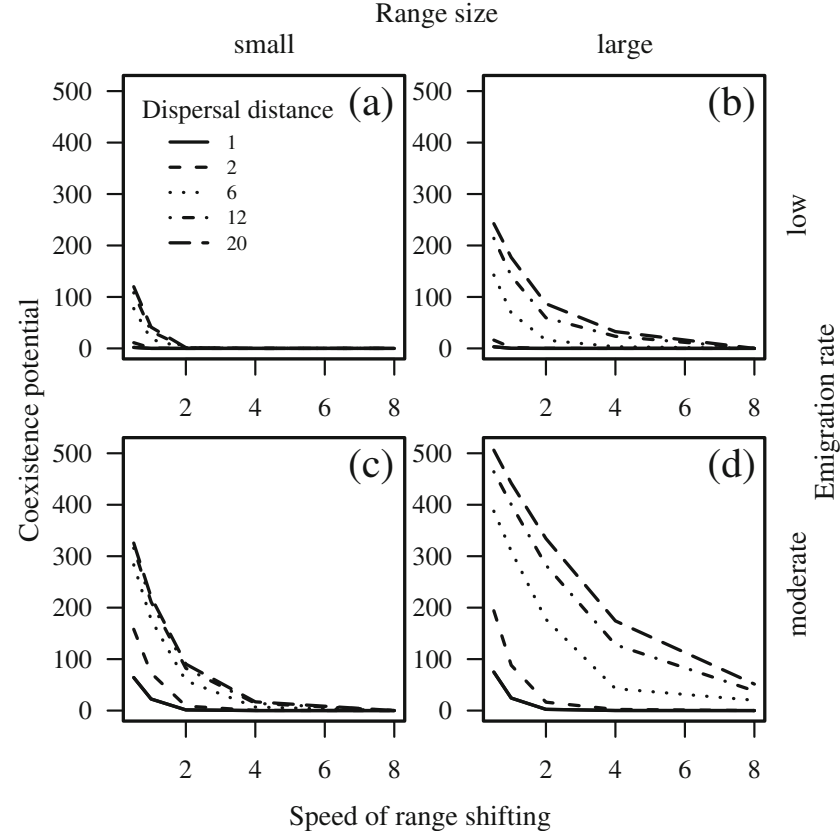

Fig. 5 Coexistence potential in dependency on speed of range shifting (shift speed) and dispersal distances. The climate window is small in the left $\left(\mathrm{CW}_{\text {size }}=20\right.$ cells, plots $\left.a, c\right)$ and larger in the right column $\left(\mathrm{CW}_{\text {size }}=\right.$ 40 cells, plots $b, d)$. Emigration rates are low in the top row $\left(p_{\mathrm{emi}}=0.01\right.$, plots $a, b)$ and moderate below $\left(p_{\text {emi }}=0.1\right.$, plots $\left.c, d\right)$

range shifting disrupted the coexistence mechanisms completely. Halving the width of the climate window reduced coexistence potential by a third (Fig. 5a and c vs. b and d) and a tenfold increase in emigration rates (from 0.001 to 0.01 ) doubled the coexistence potential (Fig. $5 \mathrm{a}$ and b vs. c and d). Thus, a strong decrease in the climate window size, i.e., in the range size, could partly be buffered by high emigration rates.

For our third research question, we investigated whether the two coexistence mechanisms responded differentially to patch isolation and range shifting (range-shifting scenario). Remarkably, the above-described general impacts of range shifting and patch isolation did not apply equally to both coexistence mechanisms: Under most conditions, coexistence potential was higher for stabilized coexistence than for neutral coexistence (Fig. 6). However, certain landscape conditions existed where only one or the other mechanism facilitated coexistence under environmental change: Only stabilized coexistence was able to cope with a combination of strong isolation and slow range shifting, and only neutral coexistence was able to follow quickly changing ranges. In general, the relative dominance of neutral coexistence increased with accelerated range shifting and decreased with decreasing dispersal distances (Fig. 6). Thus, increasing isolation had a greater impact on neutral coexistence while stabilized coexistence was more sensitive to range shifting. 


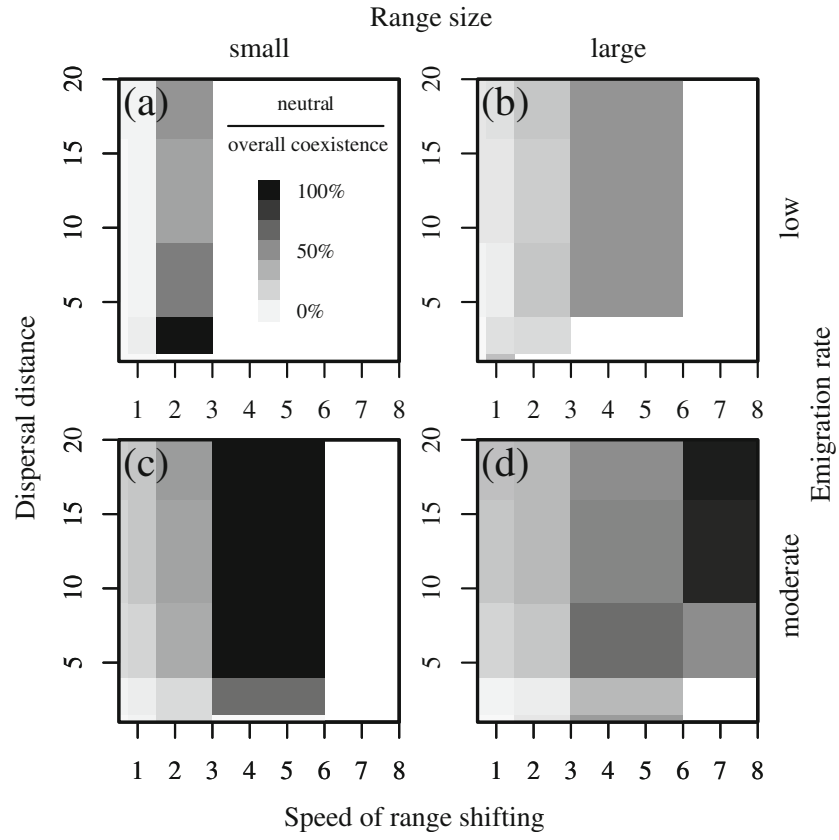

Fig. 6 Relative proportion of neutral coexistence in overall coexistence potential depending on increasing speeds of range shifting (shift speeds) and decreasing dispersal distances. The climate window is small in the left $\left(\mathrm{CW}_{\text {size }}=20\right.$ cells, plots $\left.a, c\right)$ and larger in the right column $\left(\mathrm{CW}_{\text {size }}=40\right.$ cells, plots $\left.b, d\right)$. Emigration rates are low in the top row $\left(p_{\text {emi }}=0.01\right.$, plots $\left.a, b\right)$ and moderate below $\left(p_{\text {emi }}=0.1\right.$, plots $\left.c, d\right)$. White cells indicate that overall coexistence potential equals zero

\section{Discussion}

Here, we have taken some initial steps towards understanding how the nature of the mechanisms involved in structuring communities may determine their responses to one or more environmental drivers. There has been considerable recent debate over the relative roles of stabilizing and neutral mechanisms in structuring communities and a growing body of theory addresses how these alternative mechanisms operate on temporally and spatially heterogeneous landscapes (Chesson 2000; Gotelli and Mccabe 2002; Adler et al. 2007). Our work highlights that the nature of coexistence has considerable implications for predicting and managing the consequences of environmental change on biodiversity.

Building on the framework of equalizing and stabilizing mechanisms (Chesson 2000; Adler et al. 2007), we analyze one example coexistence mechanism of each category: stabilized coexistence through overcompensation is known to work in homogeneous and stable landscapes (Münkemüller et al., submitted manuscript). However, our results demonstrate that it is not restricted to these landscapes. It also operates in fragmented landscapes if either local carrying capacities are high or patches with moderate carrying capacities are well connected (Fig. 3). High carrying capacities and/or low patch isolation are essential because stabilized coexistence depends on endogenously generated density fluctuations. Mean population densities need to be high to allow for sufficient amplitudes of these fluctuations without risking stochastic extinctions in times of density depressions. However, detrimental effects of only low to moderate carrying capacities can be buffered by low patch isolation and thus tightly coupled local population dynamics (Fig. 3c). In landscapes with a combination of low local carrying capacities and high patch isolation, the coexistence mechanism breaks down locally but as local dynamics are spatially uncorrelated (Münkemüller and Johst 2007) extinct species may re-immigrate from neighboring patches and slow down regional extinction (Fig. 3f).

The neutral mechanism only promotes long-term coexistence in fragmented landscapes with a sufficient number of weakly connected patches (Fig. 3d and f). In such landscapes, limited dispersal slows down the random drift to extinction. Recently, Wang et al. (2005) claimed that this result is only an artifact and critically depends on the assumption that extinction and colonization parameters are independent of the relative abundances of both species in commonly occupied patches. They state that this oversimplified assumption would give an unfair advantage to regionally rare species. However, our results demonstrate that coexistence does occur even with explicit consideration of local population dynamics, which automatically includes abundance-dependent local extinction and re-colonization processes.

The framework of equalizing and stabilizing mechanisms classifies coexistence mechanisms depending on dissimilarities between species (Adler et al. 2007). Our approach of coexistence along a gradient of density regulation mechanisms relates well to this framework as changes in a single species' trait (the density regulation mechanism) led from neutral to stabilized coexistence. Consequently, dissimilarity can be measured on a one-dimensional scale. With increasing dissimilarity in density regulation, neutral coexistence decreases and stabilized coexistence increases. The turnover from neutral coexistence to stabilized coexistence is gradual, with both mechanisms contributing to the overall coexistence potential at moderate species differences (Figs. 3 and 4). It has been suggested before that neutral and niche theory are not mutually exclusive (Gotelli and Rohde 2002; Gilbert et al. 2006; Chesson 2000) and that even if local species' interactions exist some properties of a community may be insensitive to these interactions and can thus be adequately described by neutral processes (Bell 2005). In this study, we demonstrate that the relative contribution of different coexistence mechanisms to overall coexistence may strongly depend on landscape configuration, i.e., patch isolation and habitat capacity: The stabilizing mechanism in this study dominates homogeneous landscapes or landscapes with large 
and strongly connected patches whereas the neutral mechanism dominates fragmented landscapes with small habitat patches. Both mechanisms co-occur in landscapes with intermediate patch isolation and patch size.

A growing body of literature demonstrates that climate change and patch isolation severely reduce species survival (e.g., Sala et al. 2000; Travis 2003; Thomas et al. 2004; Best et al. 2007). What is new in our study is the focus on community structure, i.e., on differential responses of species persisting through alternative coexistence mechanisms. Comparable to species survival, species coexistence strongly suffers from patch isolation and geographic range shifting as one possible consequence of climate change. Remarkably, already slow geographic range shifting imposes severe impacts in fragmented landscapes (Fig. 5). This result is in concordance with earlier studies demonstrating the strong effect of temporal dynamics (e.g., Fahrig 1992; Roy 2004) and implies that communities may undergo rapid changes already in initial periods of climate change leaving management actions little time to mitigate detrimental impacts. We show that, at the community level, the response to geographic range shifting and patch isolation sensitively depends on the underlying coexistence mechanisms. Communities with neutral coexistence are much more sensitive to increasing patch isolation than communities with stabilized coexistence. Although neutral communities depend on moderately isolated local population dynamics and go extinct in homogeneous or well-connected landscapes, they severely suffer from very strong isolation. This is because neutral coexistence critically relies on sufficient reimmigration to balance local drifts to extinction. Conversely, communities with stabilized coexistence are much more sensitive to range shifting than neutral communities. Stabilized communities can track even slow range shifting only if the number and size of local habitat patches are sufficiently large and well connected. The reason is that this coexistence mechanism relies on large mean population densities to buffer depressions during density fluctuations (Fig. 6). Under range shifting, however, new patches and new populations constantly need to establish when entering the climate window and are therefore on average smaller. At fast range shifting, local populations or even patches may never reach their potential size before the climate window passes. In real ecosystems, this problem becomes important when resources need a long time to establish, e.g., for species communities depending on mature forests or on upland moors. Species with small dispersal ranges and/or low emigration rates are especially vulnerable because they reach newly established patches with a delay. For them, already slow range shifting may lead to extinction. These added mortality risks reduce single-species persistence but are amplified when communities depend on well-balanced dynamics between competing species.
The results from our simple model suggest that the effectiveness of biodiversity management strategies developed to mitigate against environmental change may differ according to the coexistence mechanisms structuring a community. These results contribute to a generic understanding of some of the complex processes ruling multispecies communities. We suggest that future work focusing in three areas would further improve and complement this understanding. First, there is a clear need to validate our theoretical findings using data on real communities. Validation may be difficult to achieve when studying a single community due to the lack of decent estimates for model parameters and the multitude of different interacting dynamics but should be accessible through meta-analyses. Unfortunately, to date, there is a rather small number of field studies that have investigated the community-level responses to patch isolation or range shifting independently, let alone considering potentially synergistic effects. In general, published studies in this area confirm our finding that accelerating range shifting, e.g., due to climate change, and strong patch isolation threaten communities (Wilson et al. 2007; Echeverria et al. 2007; Manu et al. 2007; Benedick et al. 2006; Burke and Goulet 1998). Future field studies should aim to identify interactions between environmental drivers and ideally also seek to establish how communities structured by the different mechanisms may be differentially impacted. However, establishing field studies to validate the predictions of models such as that presented in this paper remains a challenging task. We agree with Benton et al. (2007) that taking a microcosm approach may offer considerable potential, at least as a complementary approach. Therefore, our second recommendation is that a number of different microcosm communities comprising a wide range of species from different taxa should be established with a view to investigating the range of community-level responses when those communities are subjected to environmental perturbations (Davis et al. 1998). As Benton et al. (2007) argued, there can be considerable benefits to be gained from replicating experiments across a range of analog model systems. In this case, it would provide an excellent opportunity to establish how well results from one community are likely to generalize across other communities. Third, further theoretical work is necessary to consider both other environmental effects, e.g., the impact of altered disturbance regimes (Easterling et al. 2000; Beniston et al. 2007; Johst and Huth 2005), and further coexistence mechanisms. These extensions would also enable us to ask new questions regarding the formation of novel assemblages during geographic range shifting with characteristics different from those under stable ranges.

An improved knowledge of the mechanisms that determine community structure has the potential to increase our understanding of how different species may respond to 
environmental change. We emphasize that we are some way from being able to construct predictive modes that incorporate this level of detail for real assemblages responding to environmental change on real landscapes. However, we believe that an improved generic understanding of the range of plausible outcomes arising due to alternative community structure has the potential to help inform the development of robust conservation strategies that can be effective regardless of the exact structuring mechanisms.

Acknowledgements TM appreciates the financial support provided by the German Federal Ministry of Education and Research (01 LB 0202). BR acknowledges funding by the European Union within the FP 6 Integrated Project “ALARM” (GOCE-CT-2003-506675).

\section{References}

Adler PB, HilleRisLambers J, Levine JM (2007) A niche for neutrality. Ecol Lett 10:95-104. doi:10.1111/j.1461-0248.2006.00996.x

Araujo MB, New M (2007) Ensemble forecasting of species distributions. Trends Ecol Evol 22:42-47. doi:10.1016/j.tree.2006.09.010

Araujo MB, Rahbek C (2006) How does climate change affect biodiversity? Science 313:1396-1397. doi:10.1126/science.1131758

Araujo MB, Cabeza M, Thuiller W, Hannah L, Williams PH (2004) Would climate change drive species out of reserves? An assessment of existing reserve-selection methods. Glob Change Biol 10:1618-1626. doi:10.1111/j.1365-2486.2004.00828.x

Bell G (2005) The co-distribution of species in relation to the neutral theory of community ecology. Ecology 86:1757-1770. doi: $10.1890 / 04-1028$

Benedick S, Hill JK, Mustaffa N, Chey VK, Maryati M, Searle JB, Schilthuizen M, Hamer KC (2006) Impacts of rain forest fragmentation on butterflies in northern Borneo: species richness, turnover and the value of small fragments. J Appl Ecol 43:967977. doi:10.1111/j.1365-2664.2006.01209.x

Beniston M, Stephenson DB, Christensen OB, Ferro CAT, Frei C, Goyette S, Halsnaes $\mathrm{K}$ et al (2007) Future extreme events in European climate: an exploration of regional climate model projections. Clim Change 81:71-95. doi:10.1007/s10584-006-9226-Z

Benton TG, Solan M, Travis JMJ, Sait SM (2007) Microcosm experiments can inform global ecological problems. Trends Ecol Evol 22:516-521. doi:10.1016/j.tree.2007.08.003

Best AS, Johst K, Münkemüller T, Travis JMJ (2007) Which species will successfully track climate change? The influence of intraspecific competition and density dependent dispersal on range shifting dynamics. Oikos 116:1531-1539. doi:10.1111/ j.0030-1299.2007.16047.x

Brooker RW, Travis JMJ, Clark EJ, Dytham C (2007) Modelling species' range shifts in a changing climate: the impacts of biotic interactions, dispersal distance and the rate of climate change. $\mathrm{J}$ Theor Biol 245:59-65. doi:10.1016/j.jtbi.2006.09.033

Burke D, Goulet H (1998) Landscape and area effects on beetle assemblages in Ontario. Ecography 21:472-479. doi:10.1111/ j.1600-0587.1998.tb00438.x

Chesson P (2000) Mechanisms of maintenance of species diversity. Annu Rev Ecol Syst 31:343-366. doi:10.1146/annurev.ecolsys.31.1.343

Davis AJ, Jenkinson LS, Lawton JH, Shorrocks B, Wood S (1998) Making mistakes when predicting shifts in species range in response to global warming. Nature 391:783-786. doi:10.1038/35842

Easterling DR, Meehl GA, Parmesan C, Changnon SA, Karl TR, Mearns LO (2000) Climate extremes: observations, modeling, and impacts. Science 289:2068-2074. doi:10.1126/science. 289.5487.2068

Echeverria C, Newton AC, Lara A, Benayas JMR, Coomes DA (2007) Impacts of forest fragmentation on species composition and forest structure in the temperate landscape of southern Chile. Glob Ecol Biogeogr 16:426-439. doi:10.1111/j.1466-8238.2007.00311.x

Etienne RS, Olff H (2005) Confronting different models of community structure to species-abundance data: a Bayesian model comparison. Ecol Lett 8:493-504. doi:10.1111/j.1461-0248.2005.00745.x

Fahrig L (1992) Relative importance of spatial and temporal scales in a patchy environment. Theor Popul Biol 41:300-314. doi:10.1016/0040-5809(92)90031-N

Ferrier S, Guisan A (2006) Spatial modelling of biodiversity at the community level. J Appl Ecol 43:393-404. doi:10.1111/j.13652664.2006.01149.x

Gause GF (1969) The struggle for coexistence. Hafner, London

Gilbert B, Laurance WF, Leigh EG, Nascimento HEM (2006) Can neutral theory predict the responses of Amazonian tree communities to forest fragmentation? Am Nat 168:304-317. doi:10.1086/506969

Godfray HCJ, Blythe SP, Williamson M, Perry JN (1990) Complex dynamics in multispecies communities. Philos Trans R Soc Lond B Biol Sci 330:221-233. doi:10.1098/rstb.1990.0194

Gotelli NJ, Mccabe DJ (2002) Species co-occurrence: a meta-analysis of J. M. Diamond's assembly rules model. Ecology 83:2091-2096

Gotelli NJ, Rohde K (2002) Co-occurrence of ectoparasites of marine fishes: a null model analysis. Ecol Lett 5:86-94. doi:10.1046/ j.1461-0248.2002.00288.x

Gravel D, Canham CD, Beaudet M, Messier C (2006) Reconciling niche and neutrality: the continuum hypothesis. Ecol Lett 9:399409. doi:10.1111/j.1461-0248.2006.00884.x

GSL Team (1992) GNU scientific library. htt9://www.gnu.org/soft ware/gsl/manual/html_node/

Guisan A, Thuiller W (2005) Predicting species distribution: offering more than simple habitat models. Ecol Lett 8:993-1009. doi:10.1111/j.1461-0248.2005.00792.x

Hassell MP, Comins HN (1976) Discrete-time models for 2-species competition. Theor Popul Biol 9:202-221. doi:10.1016/00405809(76)90045-9

Hatfield RG, LeBuhn G (2007) Patch and landscape factors shape community assemblage of bumble bees, Bombus spp. (Hymenoptera: Apidae), in montane meadows. Biol Conserv 139:150-158. doi:10.1016/j.biocon.2007.06.019

Heikkinen RK, Luoto M, Araujo MB, Virkkala R, Thuiller W, Sykes MT (2006) Methods and uncertainties in bioclimatic envelope modelling under climate change. Prog Phys Geogr 30:751-777. doi:10.1177/0309133306071957

Higgins SI, Clark JS, Nathan R, Hovestadt T, Schurr F, Fragoso JMV, Aguiar MR et al (2003) Forecasting plant migration rates: managing uncertainty for risk assessment. J Ecol 91:341-347. doi:10.1046/j.1365-2745.2003.00781.x

Hubbell SP (2001) The unified neutral theory of biodiversity and biogeography. Princeton University Press, Princeton

Hubbell SP (2006) Neutral theory and the evolution of ecological equivalence. Ecology 87:1387-1398. doi:10.1890/0012-9658 (2006)87[1387:NTATEO]2.0.CO;2

Huisman J, Weissing FJ (1999) Biodiversity of plankton by species oscillations and chaos. Nature 402:407-410. doi:10.1038/46540

Johst K, Huth A (2005) Testing the intermediate disturbance hypothesis: when will there be two peaks of diversity? Divers Distrib 11:111-120. doi:10.1111/j.1366-9516.2005.00133.x

Kruess A, Tscharntke T (1994) Habitat fragmentation, species loss, and biological-control. Science 264:1581-1584. doi:10.1126/ science.264.5165.1581

Latimer AM, Silander JA, Cowling RM (2005) Neutral ecological theory reveals isolation and rapid speciation in a biodiversity hot spot. Science 309:1722-1725. doi:10.1126/science.1115576 
Manu S, Peach W, Cresswell W (2007) The effects of edge, fragment size and degree of isolation on avian species richness in highly fragmented forest in West Africa. Ibis 149:287-297. doi:10.1111/ j.1474-919X.2006.00628.x

Maynard Smith J, Slatkin M (1973) The stability of predator-prey systems. Ecology 54:384-391. doi:10.2307/1934346

Midgley GF, Hughes GO, Thuiller W, Rebelo AG (2006) Migration rate limitations on climate change-induced range shifts in Cape Proteaceae. Div Distr 12:555-562. doi:10.1111/j.13669516.2006.00273.x

Morris WF (1990) Problems in detecting chaotic behavior in naturalpopulations by fitting simple discrete models. Ecology 71:18491862. doi: $10.2307 / 1937593$

Münkemüller T, Johst K (2006) Compensatory versus overcompensatory density regulation: implications for metapopulation persistence in dynamic landscapes. Ecol Model 197:171-178. doi:10.1016/j.ecolmodel.2006.02.041

Münkemüller T, Johst K (2007) How does intraspecific density regulation influence metapopulation synchrony and persistence? J Theor Biol 245:553-563. doi:10.1016/j.jtbi.2006.10.020

R Development Core Team (2007) R: a language and environment for statistical computing. http://www.R-project.org

Rosindell J, Cornell SJ (2007) Species-area relationships from a spatially explicit neutral model in an infinite landscape. Ecol Lett 10:586-595. doi:10.1111/j.1461-0248.2007.01050.x

Roy M, Pascual M, Levin SA (2004) Competitive coexistence in a dynamic landscape. Theor Popul Biol 66:341-353. doi:10.1016/j. tpb.2004.06.012

Sala OE, Chapin FS, Armesto JJ, Berlow E, Bloomfield J, Dirzo R, Huber-Sanwald E et al (2000) Biodiversity-global biodiversity scenarios for the year 2100. Science 287:1770-1774. doi:10.1126/science.287.5459.1770
Sinclair ARE (1989) Population regulation in animals. In: Cherrett JM, Bradshaw AD, Goldsmith FB, Grubb PJ, Krebs JR (eds) Ecological concepts: the contribution of ecology to an understanding of the natural world. Blackwell Scientific, Oxford, pp 197-242

Thomas CD, Cameron A, Green RE, Bakkenes M, Beaumont LJ, Collingham YC, Erasmus BFN et al (2004) Extinction risk from climate change. Nature 427:145-148. doi:10.1038/nature02121

Thuiller W, Brotons L, Araujo MB, Lavorel S (2004) Effects of restricting environmental range of data to project current and future species distributions. Ecography 27:165-172. doi:10.1111/ j.0906-7590.2004.03673.x

Thuiller W, Midgley GF, Hughes GO, Bomhard B, Drew G, Rutherford MC, Woodward FI (2006) Endemic species and ecosystem sensitivity to climate change in Namibia. Glob Change Biol 12:759-776. doi:10.1111/j.1365-2486.2006.01140.x

Travis JMJ (2003) Climate change and habitat destruction: a deadly anthropogenic cocktail. Proc R Soc Lond B Biol Sci 270:467473. doi:10.1098/rspb.2002.2246

Walker SC (2007) When and why do non-neutral metacommunities appear neutral? Theor Popul Biol 71:318-331. doi:10.1016/j. tpb.2006.12.008

Walker SC, Cyr H (2007) Testing the standard neutral model of biodiversity in lake communities. Oikos 116:143-155. doi:10.1111/j.2006.0030-1299.15300.x

Wang ZL, Zhang DY, Wang G (2005) Does spatial structure facilitate coexistence of identical competitors? Ecol Model 181:17-23. doi:10.1016/j.ecolmodel.2004.06.020

Wilson RJ, Gutierrez D, Gutierrez J, Monserrat VJ (2007) An elevational shift in butterfly species richness and composition accompanying recent climate change. Glob Change Biol 13:1873-1887. doi:10.1111/j.1365-2486.2007.01418.x 